This item was submitted to Loughborough's Research Repository by the author.

Items in Figshare are protected by copyright, with all rights reserved, unless otherwise indicated.

\title{
Contextual information and perceptual-cognitive expertise in a dynamic, temporally-constrained task
}

\section{PLEASE CITE THE PUBLISHED VERSION}

http://dx.doi.org/10.1037/xap0000094

\section{PUBLISHER}

(c) American Psychological Association

\section{VERSION}

AM (Accepted Manuscript)

\section{PUBLISHER STATEMENT}

This work is made available according to the conditions of the Creative Commons Attribution-NonCommercialNoDerivatives 4.0 International (CC BY-NC-ND 4.0) licence. Full details of this licence are available at: https://creativecommons.org/licenses/by-nc-nd/4.0/

\section{LICENCE}

CC BY-NC-ND 4.0

\section{REPOSITORY RECORD}

Murphy, Colm P., Robin Jackson, Karl Cooke, Andre Roca, Nicolas Benguigui, and A. Mark Williams. 2016. "Contextual Information and Perceptual-cognitive Expertise in a Dynamic, Temporally-constrained Task". Loughborough University. https://hdl.handle.net/2134/23509. 


\section{Contextual Information and Perceptual-Cognitive Expertise in a Dynamic, Temporally-}

\section{Constrained Task}

Colm P. Murphy ${ }^{1}$, Robin C. Jackson ${ }^{2}$, Karl Cooke ${ }^{3}$, André Roca ${ }^{4}$, Nicolas Benguigui ${ }^{5}$, and A. Mark Williams $^{1}$

${ }^{1}$ Brunel University London, Uxbridge, UB8 3PH, UK.

${ }^{2}$ Loughborough University, Leicestershire, LE11 3TU, UK.

${ }^{3}$ Lawn Tennis Association, National Tennis Centre, 100 Priory Lane, Roehampton, SW15 5JQ, UK.

${ }^{4}$ St Marys University, Twickenham, London, TW1 4SX, UK.

${ }^{5}$ Université de Caen Basse Normandie, Boulevard du Maréchal Juin, Campus II, F-14032, Caen Cedex, France.

Author Note

The authors would like to thank the Lawn Tennis Association for providing access to video footage and data used in test stimuli. Additionally, the authors would like to thank Julien Pansiot for creating the animation rendering engine. None of the authors have any links to companies or manufacturers who would benefit from this work. There are no conflicts of interest present.

Corresponding Author: Colm Murphy

Department of Life Sciences, Brunel University London, Middlesex, UB8 3PH, UK

Telephone: +447531696734

E-mail: colm.murphy@brunel.ac.uk

Date of submission: November 6, 2015 


\begin{abstract}
Skilled performers extract and process postural information from an opponent during anticipation more effectively than their less-skilled counterparts. In contrast, the role and importance of contextual information in anticipation has received only minimal attention. We evaluate the importance of contextual information in anticipation and examine the underlying perceptual-cognitive processes. We present skilled and less-skilled tennis players with normal video or animated footage of the same rallies. In the animated condition, sequences were created using player movement and ball trajectory data, and postural information from the players was removed, constraining participants to anticipate based on contextual information alone. Participants judged ball bounce location of the opponent's final occluded shot. The two groups were more accurate than chance in both display conditions with skilled being more accurate than less-skilled (Exp. 1) participants. When anticipating based on contextual information alone, skilled participants employed different gaze behaviors to less-skilled counterparts and provided verbal reports of thoughts which were indicative of more thorough evaluation of contextual information (Exp. 2). Findings highlight the importance of both postural and contextual information in anticipation and indicate that perceptual-cognitive expertise is underpinned by processes that facilitate more effective processing of contextual information, in the absence of postural information.
\end{abstract}

Keywords: perceptual-cognitive skills; contextual information; anticipation; skill 


\section{Contextual Information and Perceptual-Cognitive Expertise in a Dynamic, Temporally-}

\section{Constrained Task}

Researchers interested in anticipation in real-world tasks often use tennis as a vehicle to explore the underlying mechanisms due to the extreme time constraints involved. Ball speeds can reach over $200 \mathrm{~km} \cdot \mathrm{h}^{-1}$ (Gillet, Leroy, Thouvarecq, \& Stein, 2009). The time it takes for the ball to travel from one player to the other can be as little as $500 \mathrm{~ms}$, meaning it is sometimes impossible for players to respond quickly enough to return the opponent's shot based on ball flight information alone (Abernethy \& Wollstein, 1989). The extreme time constraints evident in fast ball sports (Gray, 2002a), coupled with the fact that an opponent can potentially disguise his/her intentions or present deceptive information (Jackson, Warren, \& Abernethy, 2006; Kunde, Skirde, \& Weigelt, 2011; Rowe, Horswill, Kronvall-Parkinson, Poulter, \& McKenna, 2009), suggest that the contextual information which is available prior to pertinent postural information becoming available could be both valuable and necessary when making anticipation judgments.

The majority of researchers focusing on anticipation, particularly in racket-sports, have focused on how performers use advance postural information to inform their judgments (for reviews see Crognier \& Féry, 2007; Mann, Williams, Ward, \& Janelle, 2007). However, recent calls have been made for more research investigating the role of contextual information in anticipation (CañalBruland \& Mann, 2015, Triolet, Benguigui, Le Runigo, \& Williams, 2013). It is well established that skilled performers are better able to extract and process postural information from an opponent to more accurately (e.g., Abernethy \& Russell, 1987; Jones \& Miles, 1978) and more quickly (e.g., Ward, Williams, \& Bennett, 2002; Williams, Ward, Knowles, \& Smeeton, 2002) anticipate the outcome of an upcoming event than their less-skilled counterparts. To rigorously examine how skilled performers more effectively utilize postural information, researchers have traditionally employed quasi-experimental cross-sectional designs (comparing skilled and less-skilled performers) in which participants anticipate the outcome of an event based solely on the postural information emanating from an opponent (e.g., Goulet, Bard, \& Fleury, 1989; Jackson \& Mogan, 2007). In such experimental 
set-ups, contextual information built up prior to the event is usually omitted, such that the opponent's positioning is limited to a single location of the playing area throughout trials.

However, published research suggests that skilled performers can effectively anticipate the outcome of an upcoming event based on contextual information picked up in advance of pertinent postural information becoming available (Abernethy, Gill, Parks, \& Packer, 2001; Triolet et al., 2013). Moreover, contextual information can be used by skilled performers to anticipate more effectively than when it is not available, (e.g., Crognier \& Féry, 2005, Farrow \& Reid, 2012). Several researchers (e.g., McRobert, Williams, Ward, \& Eccles, 2009; Roca, Ford, McRobert, \& Williams, 2011) have explained the processes underpinning skilled anticipation using Long-Term Working Memory (LTWM) theory (Ericsson \& Kintsch, 1995). Nevertheless, both our understanding of the extent to which performers can use contextual information to anticipate effectively in advance of pertinent postural information becoming available, and the perceptual-cognitive processes underpinning such judgments is limited.

Triolet et al. (2013) quantified anticipation behavior in professional tennis matches. A key finding of this research was that while some anticipation behavior was found to occur around the time of the opponent's racket-ball contact, a large portion of effective anticipation behavior occurred over $140 \mathrm{~ms}$ prior to the opponent striking the ball. Moreover, a large portion of this early anticipation behavior occurred when the receiving player was under 'unfavorable' conditions, that is, under extreme time constraints. If a visual-motor delay of approximately $200 \mathrm{~ms}$ (Hick, 1952) is assumed, players must have been anticipating based on information arising at least $340 \mathrm{~ms}$ prior to the opponent's racket-ball contact. Triolet and colleagues concluded that these highly skilled performers were using contextual information to anticipate in advance of pertinent postural information becoming available. These findings indicate that contextual information may play an important role in skilled anticipation, particularly in fast ball sports such as tennis, in which players often perform under extreme time constraints, and it is not viable to wait for useful postural information to become available. 
In a related study Abernethy et al. (2001) demonstrated that skilled squash players are able to anticipate shot direction and depth at levels significantly greater than chance over $580 \mathrm{~ms}$ before the opponent’s racket-ball contact. They concluded that the skilled squash players’ performance advantage was due to their superior attunement to information picked up from within their opponent's pattern of play (e.g., accurate knowledge of event probabilities, shot sequencing information). Similarly, Loffing and Hagemann (2014) demonstrated that the positioning of the opponent relative to court markings provides contextual information about the direction of the opponent's shot, and that skilled players rely more on this positional information in advance of pertinent postural information becoming available.

This body of research indicates that skilled performers can use early advance information to anticipate effectively (Abernethy et al., 2001; Triolet et al., 2013) and that they do so when placed under extreme time constraints such as when the opponent is attacking or when the distance between the two players is reduced (Triolet et al., 2013). It is not yet clear though, whether this advance information is picked up from very early occurring postural cues or from contextual information, because postural information has always been readily available to participants. While postural information from the opponent will always be available in competition as the opponent's body is always visible, the moment at which this information can reliably inform skilled performers' anticipation judgments varies across sports and situations (Abernethy et al., 2001). It is therefore difficult to infer the type of information performers are using based solely on how far in advance of the event they are acting on an anticipation judgment.

Context can be defined as "the circumstances that form the setting for an event, statement, or idea, and in terms of which it can be fully understood" (Oxford Dictionaries, 2015). Contextual information can come in many different forms, such as knowledge of an opponent's strengths/weaknesses, players’ positioning relative to one another and climatic conditions (Buckolz, Prapavesis, \& Fairs, 1988). The positive effect of providing contextual information to participants in addition to postural information has been demonstrated in several sports (for a review, see CañalBruland \& Mann, 2015). Researchers have demonstrated higher levels of anticipation performance 
when participants are provided with contextual information in the form of scores and number of balls and strikes in baseball (Paull \& Glencross, 1997), increased exposure to the bowler in cricket (McRobert, Eccles, Ward, \& Williams, 2011) and knowledge of the opponent's action preferences in soccer (Navia, van der Kamp, \& Ruiz, 2013). In tennis, Crognier and Féry (2005) demonstrated that players anticipated more accurately in a high context, which they termed high tactical initiative, condition in which they were allowed to impose their game on the opponent over a series of shots, compared to when they were not allowed to do so and no shots were played prior to the opponent's occluded passing shot. Farrow and Reid (2012) demonstrated that skilled tennis players can use knowledge of the opponent's serving tendencies to increase the speed of their anticipation judgments.

Conversely, researchers have found that presenting performers with contextual information can also yield a decrease in performance. In a simulated baseball batting task, Gray (2002a, 2002b) demonstrated that while contextual information picked up from the preceding sequence of pitches and pitch count influences batting accuracy, this information negatively affected performance when incongruence existed between the actual and expected pitch. Mann, Schaefers, and Cañal-Bruland (2014) and Loffing, Stern, and Hagemann (2015) have produced supportive findings of such an effect in handball and volleyball anticipation judgment tasks. Collectively, these findings suggest that while contextual information is often beneficial to performance, situations exist when attending to contextual information can be misleading and the use of reliable postural information may be more favorable. However, under extreme time constraints, it is not always feasible for performers to wait for such information to act.

As opposed to manipulating contextual information when postural information is readily available, we provide a novel contribution by comparing anticipation judgment accuracy either in the presence or absence of postural information at the same moment so that conclusions can be drawn about the extent to which performers can use contextual information to anticipate effectively. We consider contextual information to constitute the circumstances which form the setting for the event; circumstances which in advance, or in the absence of postural information would necessarily be relied upon to make anticipation judgments. Therefore, contextual information in our anticipation task refers 
to the sequential relative movements of the players and the ball flight in the lead up to the critical event (the to-be-anticipated shot played by the opponent), and the players' resultant positioning at the moment of the event. In contrast, postural information refers to the bodily movements of the players and their resultant racket movements. In the first experiment, we investigate the extent to which tennis players can anticipate based on contextual information alone compared with performance based on this information as well as postural information. Moreover, because skilled performers have previously been shown to anticipate more effectively than their less-skilled counterparts in advance of pertinent postural information becoming available (Abernethy et al., 2001), in the second experiment we aim to determine how they do so, by tracing the perceptual-cognitive processes underlying judgments when constrained to anticipate based on contextual information alone.

Process-tracing measures such as the recording of gaze behavior, collection of verbal reports of thoughts, and manipulations to representative tasks are often used to identify the mechanisms underpinning skilled performance (e.g., Afonso, Garganta, McRobert, Williams, \& Mesquita, 2012; Catteeuw, Gilis, Wagemanns, \& Helsen, 2010; Ward, Ericsson, \& Williams, 2013). A comprehensive understanding of the perceptual-cognitive processes underlying anticipation provides an insight into how skilled performers process information more effectively than their less-skilled counterparts. This information can then be used to guide practice organization for training and development purposes (Williams, Ford, Eccles, \& Ward, 2011).

Ericsson and Kintsch’s (1995) Long Term Working Memory (LTWM) theory states that as a result of extended experience and practice, skilled performers have developed complex domainspecific memory representations. When they encounter information during practice or competition, they can accurately encode and store this information in LTM. The information is associated with a retrieval cue in STM, meaning that when similar situations are subsequently encountered, skilled performers can rapidly access the associated information stored in LTM through these retrieval cues. Skilled performers can therefore access information about previous outcomes in similar situations to guide their anticipation judgments. During practice skilled performers are exposed to a diversity of events and situations (e.g., the opponent in a tennis practice match hitting an attacking forehand from 
inside the court), and are provided with the opportunity to respond to these events (e.g., by anticipating the depth and direction of the opponent's shot and attempting to return the shot). This gives the skilled performer the opportunity to encode information from these situations, associate this encoded information with a retrieval cue, and store the information in LTM. When skilled performers encounter a similar situation in subsequent performance, the presented information is associated with a retrieval cue, allowing for the rapid retrieval of relevant information about that situation and potentially the previous outcomes of such a situation from LTM. Less-skilled performers, on the other hand, have not developed such advanced memory representations due to their relative lack of experience. Several researchers have provided support for LTWM theory (Ericsson \& Kintsch, 1995) in domain-specific anticipation tasks (e.g., North, Ward, Ericsson, \& Williams, 2011; Roca et al., 2011; Ward, Ericsson, \& Williams, 2013), citing skilled performers more advanced domain-specific memory representations as the reason for differences in performance between skill groups.

Verbal reports of thoughts recorded during (concurrently) or immediately following (retrospectively) an anticipation task provide an insight into performers' task-specific knowledge and cognitive processes when carrying out a task (Anderson, 1987). Ericsson and Simon's (1993) protocol analysis method has frequently been used to categorize the cognitive processes underlying skilled performance. In representative domain-specific anticipation tasks, skilled performers have been shown to verbalize more thoughts relating to evaluation of the event and prediction of potential future outcomes than their less-skilled counterparts (e.g., McRobert et al., 2011; North et al., 2011; Roca et al., 2011). The higher number of evaluation and prediction statements made by skilled performers when reporting their thoughts have been interpreted as being supportive of LTWM theory (Ericsson \& Kintsch, 1995).

It has been suggested that skilled performers' more advanced memory representations in domain-specific situations direct their gaze to the more pertinent information present in the environment (Williams \& Elliott, 1999). Increasingly, researchers have collected data for perceptual and cognitive process measures in the same study so as to establish much more fully the processes contributing to performance differences (Afonso \& Mesquita, 2013; McRobert et al., 2011). While the 
visual search strategy employed is dependent on the task-constraints (Roca, Ford, McRobert, \& Williams, 2013; Williams, Janelle, \& Davids, 2004), skilled performers have usually been shown to employ different strategies to those of their less-skilled counterparts (for a review, see Mann et al., 2007).

In a representative cricket task, McRobert et al. (2011) varied the number of times in a row participants viewed a particular bowler to compare the perceptual-cognitive processes employed by batters in low- and high-context conditions. Verbal report data revealed that the batters made more evaluation and deep planning statements when contextual information was available about the bowler being viewed and skilled batters made more evaluations, predictions and deep planning statements in both conditions, which the authors suggested contributed to their superior performance on the task. Gaze data indicated that skilled batters adapted their visual search strategies in the high-context condition to more efficiently extract pertinent information from the visual display than in the lowcontext condition. These data were interpreted to mean that in high-context conditions, skilled batters are able to use contextual information (in this case picked up from an opponent's prior actions), to rapidly encode and retrieve task-relevant information stored in LTM. However, thus far, no researchers have investigated whether skilled performers use similar processes when presented with contextual information alone, as would be the case when performers are under extreme time constraints and pertinent postural information picked up from the opponent is therefore not available.

Although the aforementioned studies provide us with valuable information about perceptualcognitive expertise, to our knowledge postural information and/or incoming ball flight information has always been readily available to the participant when carrying out the task, making it possible for participants to base their judgments solely on the availability of such information. This approach may be somewhat misleading when we consider the multidimensional nature of anticipation (i.e., the potential importance and contributions of various sources of information and constraints placed on performers in the anticipation process, Müller \& Abernethy, 2012; Roca et al., 2013). Triolet et al. (2013) reported that skilled performers often act in advance of pertinent postural information becoming available due to the extreme time constraints involved. As such, it is important to determine 
how skilled performers process the information that would necessarily be relied upon when postural information is not available. In the current paper, we first investigate the extent to which skilled and less-skilled tennis players can use contextual information to anticipate. Second, we examine the perceptual-cognitive processes underlying anticipation judgments based on contextual information alone.

\section{Experiment 1}

We used a laboratory-based simulation of an anticipation task to compare the performance of skilled and less-skilled tennis players when viewing test stimuli which either omitted postural information, so as to present only the sequential relative movements of the players and the ball flight in the lead up to the opponent's shot (animated condition), or which presented this contextual information as well as postural information (normal video condition). First, we examined whether the presentation of contextual information alone allows for effective anticipation or if postural information is necessary to anticipate effectively. Based on the findings of Abernethy et al. (2001) and Triolet et al. (2013) we hypothesized that all tennis players would be able to anticipate at levels significantly greater than chance in both normal video and animated conditions. Second, we determined the extent to which providing postural information in addition to contextual information affects anticipation accuracy. We hypothesized a main effect of display such that participants would be more accurate in the normal video condition than in the animated condition due to the availability of postural information (Müller \& Abernethy, 2012). We further hypothesized a group by display interaction such that the increase in accuracy from animated to normal video condition would be greater in the skilled than the less-skilled group due to the skilled group’s greater ability to pick up and utilize postural information from an opponent (Mann et al., 2007). Third, we investigated whether skilled participants would be more accurate than their less-skilled counterparts when presented with contextual information alone, as well as when presented with both contextual and postural information. We hypothesized a main effect of group with skilled participants predicted to be more accurate than less-skilled participants in both the normal video condition in which postural information was readily available (Mann et al., 2007) and in the animated condition in which 
contextual information would be used to inform judgments (Abernethy et al., 2001; Loffing \& Hagemann, 2014).

\section{Method}

\section{Participants}

Altogether, 16 skilled ( $M_{\text {age }}=24.0$ years, $\left.S D=5.6\right)$ and 20 less-skilled $\left(M_{\text {age }}=24.1\right.$ years, $S D$ $=4.7)$ male tennis players participated. Skilled participants had a mean of $17.8(S D=5.5)$ years of tennis playing experience, 12 of whom held an Association of Tennis Professionals (ATP) singles ranking (mean career high ranking of $671[S D=418]$ in the world). Less-skilled participants had a mean of $7.0(S D=4.8)$ years of tennis playing experience. Skilled and less-skilled participants had played a mean of $13.3(S D=3.6)$ and $1.4(S D=.6)$ hours tennis per week throughout their career respectively. Skilled participants had competed in $19.1(S D=7.3)$ tournaments per year, whereas lessskilled participants had not competed in competitive tennis tournaments outside of internal staff box leagues or schools tennis. Two participants in each group were left-handed with respect to the hand they normally use to play tennis, whereas all other participants were right-handed. All participants reported normal or corrected vision and those with corrected vision wore contact lenses or glasses. Participants gave their written informed consent prior to taking part and were informed that they could withdraw at any time without penalty. The work was carried out according to the ethical guidelines of the lead university.

\section{Test Stimuli}

Footage of professional men's tennis matches was collected at the AEGON Championships (2013) at the Queens Club, London from a height of $1.9 \mathrm{~m}$ above the ground, $6.4 \mathrm{~m}$ behind the centre of the court's baseline, using a $30 \mathrm{~Hz}$ wide angle HD video camera (Contour Roam, Contour Inc., Seattle, USA). The camera was positioned such that the two players' movements and ball flight were captured with the opponent whose shot the participant was anticipating in the test stimuli always being the player positioned on the far side of the court. A total of 11 matches were recorded from various rounds of the tournament, which provided footage of 15 different players. 
Test stimuli were created in two display conditions, namely normal video and animated (see Figure 1). Animated trials were created using player movement and ball trajectory information of the same points used in the normal video condition. A 10-camera system positioned around the court during matches tracked the ball’s trajectory and players' movements throughout points (Hawk-Eye Innovations Ltd., Basingstoke, England). Ball flight information was in the form of trajectory data (x, $\mathrm{y}, \mathrm{z}$ coordinates as a function of time) while player movement data were in the form of $\mathrm{x}, \mathrm{y}$ coordinates. The data from selected points were input into the LTA rendering engine (Julien Pansiot, London, UK, 2013) through which animated videos of the data were generated for use with VLC media player (VideoLAN, Paris, France). The principle difference between the two display conditions was that postural information of the players was not visible in the animated condition. In place of seeing the players and their rackets, participants saw a blue and a red cylinder representing each player and the ball as a yellow dot, while rackets were not visible. In both display conditions trials were occluded at the opponent's racket-ball contact using Pinnacle Studio 15 editing software (Pinnacle, Ottawa, Canada).

$<<$ Insert Figure 1 here $>>$

Experimental trials were made up of footage from 90 rallies, with the final occluded shot played by the opponent to one of four areas on the court. Those shots the opponent hit on which the first bounce landed before or after the service line were classified as short and deep respectively, while those shots which landed to the right or left of the centre line were classified as such. The number of shots played to the four areas of the court on the final occluded shot was: deep-left (32); deep-right (32); short-left (13); and short-right (13). The order of the bounce location of the opponent's final occluded shot was randomized across trials and conditions. The number of shots played prior to the opponent's occluded shot in trials was between 2 and 12. Trials were between 5 and 18 seconds long, beginning 3 seconds prior to racket-ball contact of the serve at the beginning of the point. An inter-trial interval of 6 seconds was employed. 
Three Lawn Tennis Association (LTA) tennis coaches, all of whom were coaching national and/or international players on a full-time basis, recommended presenting situations in which the receiving player (on the near side of the screen) was placed under extreme time constraints as test stimuli. The rationale for doing so was that in a match situation such extreme time constraints are likely to force the player to anticipate the outcome of the opponent's shot early, in order to return it successfully, as it may not be feasible to wait for postural or ball flight information to become available. In support of this practical recommendation, Triolet et al. (2013) reported that in professional men's tennis matches, anticipation behavior is more prevalent when the balance of power is unfavorable for the receiving player, and when the spatiotemporal constraints are therefore more extreme. The selected situations in which trials were occluded at the opponent's racket-ball contact were made up of attacking forehand and backhand drives, passing shots, volleys, and drop-shots, all of which meant that the receiving player was under extreme time constraints, the balance of power was unfavorable for him, or both. A total of 150 points (150 videos and the corresponding 150 data sets to be used to render animations) were originally selected, with points being omitted if the footage was of low quality, due to poor weather and lighting, or if the data used to render the animations contained errors, such as gaps in ball flight information. We thereby reduced the number of trials from 150 to 90, which were viewed in both normal video and animated display conditions (90 normal videos and 90 animated trials).

\section{Materials, Apparatus, and Set-Up}

Footage was projected on to a $4.1 \times 2.3 \mathrm{~m}$ white projection screen (AV Stumpfl, Wallern, Austria) using a NEC PE401H HD projector (NEC, Tokyo, Japan). Participants began each trial standing $5 \mathrm{~m}$ from the screen holding a racket in their hands as if ready to play a point. Testing was conducted in a large enough room for participants to be able to move around and swing the racket freely. The screen further allowed for recreation of a realistic vertical visual angle of $2.5^{\circ}-3.7^{\circ}$ subtended by the opposing player. A similar visual angle has been used in other studies of this nature, (e.g., Loffing, Wilkes, \& Hagemann, 2011). 


\section{Procedure}

Participants viewed 16 familiarization and 180 experimental trials, half of which were shown in the normal video condition and half in the animated display condition. Participants viewed trials in blocks of 30 with a one minute break between each of the blocks except between the third and fourth blocks for which they had a three minute break (merely to have a longer break half-way through the protocol). The order of display conditions was counterbalanced across participants: half the participants in each group viewed a block of 30 animated trials followed by five further blocks of 30 clips that alternated between normal video and animated trials. The remaining participants viewed a block of 30 normal trials followed by five further blocks of 30 trials that alternated between animated and normal video. Prior to commencing the protocol, participants were informed that each of the occluded shots played by the opponent landed in the legal playing area, in one of the four sections of the court (deep-left, deep-right, short-left, short-right). During trials participants were allowed to move freely. Upon occlusion at the opponent's racket-ball contact, the screen would go black and participants verbally indicated depth and direction of the ball bounce location, while swinging the racket and moving as if to return the shot. The verbal response was recorded.

\section{Data Analysis}

Judgment accuracy was reported as the percentage of correct anticipation judgments relative to the actual final ball bounce location of the occluded shots. To determine whether participants could anticipate more effectively than chance, paired samples $t$-tests were carried out to compare depth, direction, and combined judgment accuracy of the two groups with judgment accuracy that would be expected due to chance. To examine the extent to which skilled and less-skilled participants can use contextual information alone and both contextual and postural information to inform their judgments, a $2 \times 2$ (Display [normal video, animated] $\times$ Group [skilled, less-skilled]) multi-factorial MANOVA with repeated measures was conducted for judgment accuracy, with the percentage of correct depth, direction, and combined judgments serving as the dependent variables. Wilks' Lambda values are reported for the multivariate output while the Greenhouse-Geisser correction was applied in the case 
of violations of Mauchly's test of sphericity. Partial eta squared $\left(\eta_{\mathrm{p}}{ }^{2}\right)$ values are reported throughout for effect size of main effects. The alpha level of statistical significance was set at .05 with the sequential Bonferroni correction applied to control for family-wise error where multiple $t$-test comparisons were conducted. Finally, pairwise comparisons were carried out in the case of significant interactions while Cohen's $d$ is reported for effect size of these comparisons.

\section{Results and Discussion}

\section{Judgment Accuracy Data}

Mean scores and standard errors of judgment accuracy for the skilled and less-skilled groups are presented in Figure 2. First, we hypothesized that participants would be more accurate than chance in both the normal video and animated conditions. As expected, both groups' judgment accuracy scores were significantly higher than would be expected due to chance in both normal video and animated display conditions when making depth, direction, and combined anticipation judgments (all $p<.001)$. This primary finding demonstrates that tennis players can use contextual information to make more accurate anticipation judgments than would be expected due to chance when no postural information is available from the visual display to inform their judgments. This finding supports previous research showing that contextual information plays an important role in anticipation (Abernethy et al., 2001; Triolet et al., 2013). Moreover, data extend previous work by confirming that anticipation judgments may be made more accurately than would be expected due to chance in the absence of pertinent postural information. The high mean scores for combined judgments of both skilled and less-skilled groups in the animated condition (49.10\% and $44.00 \%$ respectively) when compared to chance level (25.00\%) indicate that not only is this a useful source of information, but it is a source that both skilled and less-skilled performers are able to exploit. These findings resemble those in studies in which participants have been presented with point-light displays of the opponent alone. In that body of research, performers were shown to be able to use minimal biological motion information to anticipate at levels significantly greater than chance (e.g., Abernethy, Zawi, \& Jackson, 2008; Abernethy \& Zawi, 2007; Ward, et al., 2002). In the present experiment, the sequential relative 
movement of the players and the ball flight provided a sufficiently informative basis from which participants were able to judge the outcome at better than chance levels.

We hypothesized that participants would be more accurate in the normal video than in the animated condition. Analysis of the judgment accuracy data was consistent with this hypothesis, revealing a significant multivariate main effect for Display, Wilks’ Lambda $=.06, F(3,32)=166.73$, $p<.001, \eta_{\mathrm{p}}{ }^{2}=.94$. The univariate output revealed a significant main effect of Display for the depth, $F(1,34)=490.52, p<.001, \eta_{\mathrm{p}}{ }^{2}=.94$, direction, $F(1,34)=14.05, p=.001, \eta_{\mathrm{p}}{ }^{2}=.29$, and combined judgment data, $F(1,34)=110.09, p<.001, \eta_{\mathrm{p}}{ }^{2}=.76$. For all judgment measures mean scores were higher in the normal video condition than in the animated condition. From this it can be inferred that while contextual information enables performers to anticipate well above chance level, postural information makes a significant additional contribution to anticipation skill.

Reflecting the above effect sizes, the advantage of viewing normal video as opposed to the animation was greater for depth judgment accuracy (normal: 82.19\%, animated: 67.38\%) than for directional judgments (normal video: 68.89\%, animated: 65.89\%). This suggests that postural information may be particularly important for anticipating depth. For example, the most useful cues when anticipating drop shots may emerge from the angle and speed of the racket head on approach to racket-ball contact. Abernethy et al. (2008) showed that when players had to anticipate a smash or drop shot in badminton while viewing a point-light display showing only the shuttle and either the racket, the arm, the upper body, or the lower body, the most informative source at racket-shuttle contact for the skilled players was the racket.

We further hypothesized that the increase in accuracy from the animated to the normal video condition would be more pronounced in the skilled than the less-skilled group, and that skilled participants would be more accurate than their less-skilled counterparts in both normal video and animated conditions. The MANOVA revealed a significant main effect of Group, Wilks' Lambda = $.60, F(3,32)=7.08, p=.001, \eta_{\mathrm{p}}{ }^{2}=.40$, with the univariate analysis indicating a significant group effect for the depth, $F(1,34)=7.88, p=.008, \eta_{\mathrm{p}}{ }^{2}=.19$, direction, $F(1,34)=11.21, p=.002, \eta_{\mathrm{p}}{ }^{2}=$ 
.25 , and combined judgment data, $F(1,34)=21.76, p<.001, \eta_{\mathrm{p}}{ }^{2}=.39$. In each case, skilled participants were more accurate than their less-skilled counterparts. The hypothesised Display $\times$ Group interaction approached significance overall, Wilks’ Lambda $=.81, F(3,32)=2.43, p=.083$,

$\eta_{\mathrm{p}}{ }^{2}=.19$, with the univariate analysis revealing a significant Display $\times$ Group interaction for direction judgments, $F(1,34)=7.07, p=.012, \eta_{\mathrm{p}}{ }^{2}=.17$ (see Figure 2 ). To clarify the source of these findings pairwise comparisons revealed significantly higher accuracy for the skilled group for depth (normal video: $d=1.06, p=.002$; animated: $d=0.70, p=.023$ ) and combined (normal video: $d=1.72, p<$ .001 ; animated: $d=0.93, p=.005$ ) judgments across both display conditions, while for direction judgments the group effect was significant and large in the normal video condition $(d=1.36, p<$ $.001)$, but smaller and non-significant in the animated condition ( $d=0.51, p=.073)$. Collectively, these findings confirm that skilled players: a) use postural information picked up from an opponent to anticipate more effectively than their less-skilled counterparts; (Mann et al., 2007) and b) can use contextual information more effectively than less-skilled participants in the absence of postural information.

$<<$ Insert Figure 2 here $>>$

To summarize, this experiment demonstrated that both skilled and less-skilled tennis players are able to use contextual information to anticipate more effectively than would be expected due to chance when postural information is not available. This finding is important because tennis players are often required to anticipate in advance of pertinent postural information becoming available due to extreme time constraints (Triolet et al., 2013). Furthermore, findings indicate that while both skilled and less-skilled players can use contextual and postural information to anticipate more effectively than would be expected due to chance, both groups can use postural information to anticipate more accurately when it is available than when it is not. Moreover, skilled players can use contextual information to anticipate more effectively than less-skilled players in the absence of postural cues. The findings of this experiment alone however, are not sufficient to understand how skilled performers process contextual information to anticipate more effectively than their less-skilled counterparts. 


\section{Experiment 2}

In Experiment 2, we trace the perceptual-cognitive processes underlying anticipation when constrained to anticipate based on contextual information alone. To understand the processes underpinning skilled anticipation performance, researchers have reproduced the conditions of validated representative anticipation tasks, and collected verbal report and gaze data to aid their understanding of how skilled performers perceive and process information differently to less-skilled performers (e.g., McRobert et al., 2011; North et al., 2011; Roca et al., 2011).

In anticipation tasks in sports other than tennis, when asked to report their thoughts concurrently or retrospectively, skilled performers have been shown to make more verbal statements which are indicative of advanced domain-specific memory representations which facilitate the retrieval and utilization of task-specific information from LTM (e.g., McRobert et al., 2009; North et al., 2011). However, no researchers have investigated the processes underpinning skilled performance when constrained to anticipate based on contextual information alone. It is important to note that researchers have demonstrated that tennis players can verbalize their thoughts during performance providing an indication of the information they are attending to (McPherson, 1999, 2000; McPherson \& Kernodle, 2007). This body of work, investigating the cognitive processes underpinning skilled performance in tennis, has shown that during competition, skilled players' verbal reports are more detailed and varied than less-skilled players and has provided an indication of the information players attend to during competition. It is also evidence of the utility and effectiveness of verbal reports as a source of data.

Williams and Davids (1997) originally collected verbal reports of thoughts in conjunction with eye-movement data to verify the information participants were attending to in a soccer anticipation task, finding that the relationship between visual fixation and the information participants verbally reported they were attending to, was dependent on the nature of the task. In a task which evoked the use of foveal vision for information extraction, the area of the display fixated on was also verbally reported by participants, whereas when information was extracted through the use of 
peripheral vision, participants reported attending to different information to that which they were fixating on during the task.

Skilled performers have been shown to exhibit different gaze behaviors to less-skilled performers (e.g., Ward et al., 2002; Williams et al., 2002). While there is a large body or research evidence to indicate that skilled performers use less fixations of longer duration when searching for information in some tasks (Mann et al., 2007), some researchers interested in anticipation in tennis have observed no differences in search rate (numbers of fixations and fixation locations, fixation duration) between high and low-skilled players but instead differences have been demonstrated in the time players spend fixating on various sources of information (Ward et al., 2002; Williams et al., 2002). It has however been recommended that gaze data should be interpreted with caution, as they merely indicate the location of the fovea during task performance, without assessing the potentially important role played by peripheral vision (Mann \& Savelsbergh, 2015). In some situations it has been suggested that skilled performers merely adopt an appropriate point upon which to anchor foveal vision. This strategy is thought to accommodate attending to, and processing of task relevant information picked up from other pertinent areas of the environment through the use of peripheral vision (Ripoll, Kerlirzin, Stein, \& Reine, 1995; Vaeyens, Lenoir, Williams, Mazyn, \& Philippaerts, 2007; Williams \& Elliott, 1999). In such instances, researchers can only make inferences as to what information participants attend to, highlighting the need to collect more direct measures such as verbal reports of thoughts in conjunction with gaze data (Williams \& Davids, 1998).

In this experiment, we compared skilled and less-skilled tennis players’ ability to anticipate shot direction and depth when viewing test stimuli in the animated condition, while collecting eyemovement data during trials and immediate retrospective verbal reports of thoughts following selected trials. We used the animated condition as opposed to the normal video condition because we are interested in the perceptual-cognitive processes underlying anticipation based on contextual information alone, rather than when postural information is available. We hypothesized, based on the findings of Experiment 1, that both groups would be able to anticipate at levels significantly greater than chance and that the skilled participants would record higher judgment accuracy scores than their 
less-skilled counterparts. As skilled participants appear to be able to use contextual information picked up from the display to anticipate more effectively than less-skilled participants, we further hypothesized that the skilled participants in this experiment would process such information differently to less-skilled participants. Based on LTWM theory (Ericsson \& Kintsch, 1995), as well as previous research on anticipation (McRobert et al., 2011; North et al., 2011; Roca et al., 2011), we hypothesized that skilled participants would make more evaluation and prediction statements than less-skilled participants. This finding would be indicative of the skilled participants' more advanced domain-specific memory representations. We further analyzed participants' verbal reports based on domain-specific keywords to provide an indication of the information participants are consciously attending to when presented only with contextual information. We hypothesized that skilled participants’ verbal reports would be more detailed and varied than less-skilled participants’ (McPherson, 1999, 2000; McPherson \& Kernodle, 2007), which would be demonstrated by their use of a greater amount of different keywords than less-skilled participants. Finally, based on previous research on anticipation in tennis, we hypothesized that the skilled and less-skilled participants' search rate would not differ but rather that differences would be observed in the amount of time participants fixate on the various features of the display (Ward et al., 2002; Williams et al., 2002).

\section{Method}

\section{Participants}

A total 10 skilled $\left(M_{\text {age }}=28.6\right.$ years, $\left.S D=4.7\right)$ and 10 less-skilled $\left(M_{\text {age }}=23.7, S D=4.4\right)$ male tennis players participated in this experiment. Six of the skilled participants and four of the lessskilled participants had taken part in Experiment 1. The time period between the two experiments was approximately 10 to 12 months. Skilled participants had a mean of $22.0(S D=5.3)$ years of playing experience, five of whom held an Association of Tennis Professionals (ATP) singles ranking (mean career high ranking of $788[S D=559]$ in the world $)$. Less-skilled participants had a mean of $3.8(S D=$ 3.9) years of playing experience. Skilled participants had played tennis for a mean of $14.5(S D=4.2)$ hours per week throughout their career and competed in a mean of $19.2(S D=11.9)$ competitions per 
year, whereas less-skilled participants had played a mean of $1.6(S D=1.5)$ hours per week, and had not competed in competitive tournaments outside of schools tennis. One participant in the skilled group and two participants in the less-skilled group were left-handed with respect to the hand they normally use to play tennis with, whereas all other participants normally use their right hand. As in Experiment 1, participants gave their informed consent to take part and were informed that they could withdraw at any time without penalty. The research was carried out according to the ethical guidelines of the lead university.

\section{Test Stimuli}

A total of 28 trials in the animated display condition, which were shown to positively discriminate between skilled and less-skilled participants in Experiment 1, were used as test stimuli. The 28 trials were made up of 8 familiarization and 20 experimental trials. In Experiment 1, skilled participants had recorded combined judgment accuracy scores at least $8.75 \%$ more accurate than lessskilled participants for each of the 20 experimental trials used. The number of shots played prior to the opponent's occluded shot in trials was between 2 and 12. Trials lasted between 5 and 18 seconds including a still frame that was presented for three seconds prior to the racket-ball contact of the serve at the beginning of the point.

In experimental trials the number of shots played to the four areas of the court on the final occluded shot were: deep-left (8); deep-right (8); short-left (2); and short-right (2). The order of the bounce location of the opponent's final occluded shot was randomized throughout trials. An inter-trial interval of 6 seconds was employed in all trials except the eight trials in which participants were required to provide an immediate retrospective verbal report, with the inter-trial interval being extended until participants had finished reporting their thoughts in those trials.

\section{Materials, Apparatus, and Set-Up}

The experimental set-up and visual angle employed was the same as in Experiment 1. In addition, participants' gaze was recorded using a Mobile Eye eye-tracking system (Applied Science Laboratories, Bedford, MA, USA) during trials. The head-mounted system is integrated in a pair of 
glasses connected to a digital transmission unit, which is wirelessly connected to the recording device and worn by the participant in a small backpack. The gaze recording system records two images with two separate cameras; an image of the participant's eye and the scene image. The system integrates these two images to create one video recording of the scene video with a superimposed gaze cursor. The gaze data were analyzed using ASL Results Plus (Applied Science Laboratories, Bedford, MA, USA). The system is accurate to $\pm 1^{\circ}$ visual angle, with a horizontal and vertical precision of $1^{\circ}$.

Verbal reports of thoughts were recorded using a lapel microphone, a compact diversity receiver, a body-pack transmitter (ew112-p G3; Sennheiser, Wedemark, Germany) and a hand-held recording device (Zoom H5; Zoom Corporation, Tokyo, Japan).

\section{Procedure}

Prior to commencing testing, participants were given a brief overview of the experimental protocol. The microphone was attached to the lapel of the participant and the body-pack transmitter to their belt-strap. Participants then took part in between 20 and 35 minutes verbal report training on how to provide retrospective verbal reports of thoughts to ensure participants provided only level 1 and 2 verbalizations (based on Ericsson \& Kirk (2001) using an adaptation of Ericsson \& Simon’s (1993) protocol). According to Ericsson and Simon (1980), Level 1 verbalizations are reports of heeded information, Level 2 verbalizations are reports of information that was heeded but is in some mode other than verbal, such as visual information. Ericsson and Simon (1980) stated that verbal reports should only be made up of Level 1 and 2 verbalizations as the researcher is interested in information processed during the task as opposed to reports of information that is not heeded during the task (Level 3 verbalizations) and is therefore not reported as a result of the cognitive processes underlying performance. The training consisted of instructions on how to report thoughts retrospectively, description of how providing verbal reports differs to normal conversation, and practice providing retrospective verbal reports on generic tasks (for details on training participants to provide valid verbal reports of thoughts, see Eccles, 2012). The training was designed to encourage participants to only report Level 1 and 2 verbalizations through feedback about the verbal reports they 
gave during these tasks. Participants were encouraged to ask questions throughout the training and were provided with good and bad examples and the difference between such examples, of retrospective verbal reports based on these tasks.

Following the verbal report training, participants were fitted with the gaze recording system, placing the eye-tracking glasses on their head and the digital transmission unit in a small backpack on their back. The system was calibrated using seven non-linear calibration points presented as a grid on the visual display which encompassed the entire area of the display participants could potentially fixate on. This procedure was undertaken to ensure that the participants' point of gaze was accurately recorded. Calibration of the system was checked prior to starting the familiarization trials, between familiarization and experimental trials, and periodically during testing.

Following calibration of the gaze recording system, participants viewed eight familiarization trials from a distance of $5 \mathrm{~m}$. Trials were occluded at the opponent's racket-ball contact, at which point participants responded in the same way as in Experiment 1. Participants were then asked to provide an immediate retrospective verbal report of their thoughts following each of the familiarization trials. The same procedure was in place for the 20 experimental trials; however participants only provided a verbal report of their thoughts when asked to, following eight randomly ordered trials (the eight most discriminating trials from Experiment 1). All participants provided verbal reports on the same eight trials. The testing protocol was completed within 60 minutes.

\section{Data Analysis}

\section{Judgment accuracy data}

The number of correct trials out of twenty was expressed as a percentage with respect to depth, direction, and combined judgments. Paired samples $t$-tests were conducted to compare judgment accuracy scores of the two groups with what would be expected due to chance. Independent $t$-tests were conducted to determine differences in judgment accuracy between groups with percentage of correct depth, direction, and combined judgments serving as dependent variables. 
Verbal report data

The verbal reports provided in the experimental trials were transcribed and coded using Ericsson and Simon’s (1993) protocol analysis method, further developed by Ward, Williams, and Ericsson (2003). Reports were initially segmented using natural speech and other syntactical markers. Reports were then coded as being made up of three statement types: monitoring statements which were coded as all statements in which participants recalled current actions or events; evaluation statements, which were all statements in which participants assessed the situation relative to a specific event; and prediction statements, which were all statements in which anticipation of future or potential future events was evident (Ericsson and Simon, 1993). These three categories were used due to their use in previous studies on anticipation using verbal reports. Moreover, these categories have been successfully employed to interpret the cognitive processes underpinning expertise. Using the same coding system would therefore allow us to compare our findings with those of other related studies. Pairwise comparisons were first conducted to determine whether there was a difference in the length of verbal reports (number of words) made by skilled and less-skilled participants. This procedure was undertaken to ensure that any differences were due to the quality and/or type of participants' reports as opposed to the length of their reports. Next, to examine the type of verbal statements used by skilled and less-skilled participants, a $2 \times 3$ (Group [skilled, less-skilled] $\times$ Statement Type [monitoring, evaluation, prediction]) ANOVA was conducted, with Group as the between-participant factor and Statement Type as the within-participant factor. Finally, pairwise comparisons were conducted to investigate differences between participants in the type of statement made.

To conduct an initial investigation of what sources of contextual information participants were using from the display to inform their judgments a bespoke domain-specific keyword coding/categorization system was developed. Roca et al. (2013) used a similar approach when identifying interactions between the perceptual-cognitive skills underlying anticipation in soccer. Keywords were broken down into six categories: player positioning keywords which referred to the movement and positioning of the players; court geometry referring to the angles and spaces presented 
between players; shot type referring to any type of shot used in tennis (e.g., forehand/lob); ball flight referring to the speed, spin, and height of shots hit; shot placement referring to the depth and direction of a players' shot; and balance of power referring to the tactical situation within the rally. Once keywords were coded within reports, the percentage of participants' reports containing each of these keywords was calculated. A $2 \times 6$ (Group $\times$ Keyword Type [player positioning, court geometry, shot type, ball flight, shot placement, balance of power]) ANOVA was conducted, with Group as the between-participant factor and Keyword Type as the within-participant factor.

Gaze data

Search rate data were made up of the mean number of fixations, mean number of fixation locations, and mean fixation duration. A fixation was defined as the participant's point of gaze staying stationary for three frames or more $\left(>/=100 \mathrm{~ms}\right.$ ) within $1.5^{\circ}$ of movement tolerance (see Williams \& Davids, 1998). To determine any between-group differences, independent $t$-tests were conducted for search rate, with mean number of fixations, mean number of fixation locations, and mean fixation duration as the dependent variables and Group as the independent variable.

Percentage viewing time was defined as the portion of time the participant spent fixating on a particular area of interest. Areas of interest were: the (receiving) player; the opponent; the ball flight; the near side of the court; the far side of the court; and the play area (any other area of the display within which the ball could potentially travel throughout the trial); and unclassified areas.

Unclassified fixations were subsequently omitted from the analysis as they made up less than $1 \%$ of total fixation time. Ball flight as opposed to the ball alone was used as an area of interest. The ball flight area of interest subtended a visual angle of $6.0^{\circ}$ in the direction the ball was travelling and $1.2^{\circ}$ in the corresponding perpendicular plane, thus encompassing when participants were looking at the ball as well as if they made a visual saccade to where the ball was going to bounce or be played to (Croft, Button, \& Dicks, 2010; Singer et al., 1998), and if participants’ gaze lagged behind the ball (Land \& McLeod, 2000). The size of the shape used to identify when participants' gaze was on ball flight was maintained constant throughout the analysis. To examine the amount of time skilled and 
less-skilled participants spent viewing the various areas of the visual display, a $2 \times 6$ (Group $\times$ Fixation Location [player, opponent, ball flight, near side, far side, play area]) ANOVA was conducted with Group as the between-participant factor and Fixation Location as the withinparticipant factor.

The first author analyzed all trials, and conducted further analysis to determine intra-observer reliability one week later. Inter-observer agreement was conducted by an independent investigator. Intra- and inter-observer reliability for verbal report and gaze data ranged from $84 \%$ to $93 \%$ (see Thomas, Nelson, \& Silverman, 2005 for procedures used to determine intra- and inter-observer reliability).

The Greenhouse-Geisser correction was employed in the case of violations of Mauchly's test

of sphericity. Partial eta squared $\left(\eta_{\mathrm{p}}{ }^{2}\right)$ values are reported for effect size of main effects and the alpha level of statistical significance for all tests was set at .05 with the sequential Bonferroni correction applied to control for family-wise error where multiple $t$-test comparisons were conducted. Pairwise comparisons were carried out in the case of significant interactions and main effects. Cohen's $d$ is reported for effect size of these comparisons.

\section{Results and Discussion}

\section{Judgment Accuracy Data}

We hypothesized, based on the findings of Experiment 1, that both skilled and less-skilled participants would anticipate at levels significantly greater than chance, and that the skilled participants' judgments would be more accurate than the less-skilled participants'. Mean percentage scores and standard errors for judgment accuracy of both skilled and less-skilled groups are presented in Figure 3. Both skilled and less-skilled participants’ judgment accuracy scores for depth, direction, and combined judgments were significantly higher than would be expected due to chance $(p<.001)$. This finding reaffirms the ability of tennis players to use contextual information to anticipate effectively when postural information is not available, signifying the important role this form of information plays in anticipation (Abernethy et al., 2001; Triolet et al., 2013). This finding is 
particularly important due to the extreme time constraints experienced during fast ball sports such as tennis which mean it may not always be feasible for players to wait for pertinent postural information to become available. Skilled participants were significantly more accurate than less-skilled participants when making depth $(d=1.59, p=.001)$ and combined $(d=1.86, p<.001)$ but not direction $(d=0.32, p=.202)$ judgments.

$<<$ Insert Figure 3 here $>>$

\section{Verbal Report Data}

\section{Statement Type}

First, pairwise comparisons revealed no differences between the number of words contained in skilled and less-skilled participants' verbal reports $(d=0.27, p=.279)$. Any differences that may be found between or within groups are therefore a result of the quality or type of report provided as opposed to the report length. We hypothesized, based on LTWM theory (Ericsson \& Kintsch, 1995) and previous research on anticipation (McRobert et al., 2011; North et al., 2011; Roca et al., 2011), that skilled participants would make more evaluation and prediction statements than their less-skilled counterparts. Mean number of statements and standard errors are presented in Figure 4. There was no significant Group $\times$ Statement Type interaction, $F(1.22,22.01)=.60, p=.480, \eta_{\mathrm{p}}{ }^{2}=.03$, however a main effect of Statement Type was found, $F(1.22,22.01)=4.60, p=.037, \eta_{\mathrm{p}}{ }^{2}=.20$. No main effect of Group was observed, $F(1,18)=.53, p=.478, \eta_{p}{ }^{2}=.03$. Pairwise comparisons for Statement Type revealed that participants made significantly more monitoring $(M=10.10, S E=2.09)$ and prediction $(M=6.6, S E=.54)$ than evaluation $(M=4.25, S E=.68)$ statements $(d=0.86, p=.039$ and $d=0.81$, $p=.022$ respectively). To test our a priori prediction that the skilled group would use more evaluation and prediction statements than the less-skilled group, we conducted two planned contrasts. In support of Ericsson and Kintsch’s (1995) LTWM theory, the first of these revealed significant differences between skilled $(M=5.60, S E=.87)$ and less-skilled groups $(M=2.9, S E=1.04)$ for evaluation statements ( $d=0.89, p=.031)$, while the difference between skilled $(M=7.4, S E=.52)$ and lessskilled $(M=5.8, S E=.94)$ groups for prediction statements was not significant, $(d=0.66, p=.077)$. 
Findings indicate that when presented with only contextual information, skilled participants appear to evaluate the situation and potential outcomes more effectively than their less-skilled counterparts. This effect potentially contributes to their more accurate anticipation judgments when constrained to anticipate based on contextual information alone.

Our findings are in support of previous research which has shown that skilled performers make more statements which are indicative of more advance memory representations, that is more evaluation statements. Although the difference between groups in the amount of prediction statements made did not reach statistical significance, the observed effect size was medium to large. We therefore suggest that similar cognitive processes underlie skilled anticipation when constrained to use contextual information alone as when postural information is readily available for processing (McRobert et al., 2009; North et al., 2011; Roca et al., 2011). Skilled participants are likely to have regularly encountered similar situations and as such may have developed advanced memory representations from which they can access information for evaluative purposes. When skilled participants perceive contextual information in the form of sequential relative movements of the players and the ball flight in the lead up to the event, it is possible that some feature(s) picked up from the display, may act as a retrieval cue, which is activated to retrieve task-relevant information from LTM. This information potentially helps guide participants' judgments by allowing for more thorough evaluation of the event, and prediction of potential event outcomes, relative to previously experienced situations. While previous research on anticipation has always presented participants with postural information which could act as the retrieval cue needed to anticipate more effectively, our findings indicate that skilled performers may be able to use some form of contextual information as a retrieval cue to access pertinent task relevant information from LTM during anticipation.

$<<$ Insert Figure 4 here $>>$

Keyword Type

Based on the research of McPherson and colleagues (McPherson, 1999; McPherson, 2000; McPherson \& Kernodle, 2007), we hypothesized that skilled participants’ verbal reports would be 
more detailed and varied than less-skilled participants'. To analyze the Keyword Type data we first calculated the percentage of participants' reports containing each type of keyword. These data are presented in Figure 5. ANOVA revealed the Group $\times$ Keyword Type interaction did not reach statistical significance, $F(2.92,52.62)=2.46, p=.074, \eta_{\mathrm{p}}{ }^{2}=.12$, however significant main effects of Group, $F(1,18)=5.99, p=.025, \eta_{\mathrm{p}}{ }^{2}=.25$ and Keyword Type, $F(2.92,52.62)=19.56, p<.001, \eta_{\mathrm{p}}{ }^{2}=$ .52, were found. The main effect of Group indicates that, in line with our hypothesis, skilled participants used a greater number of different keywords per report $(M=2.73, S E=.22)$ than lessskilled participants $(M=1.94, S E=.24)$.

Further independent $t$-tests revealed that significantly more of the skilled participants' reports contained court geometry $(d=1.28, p=.005)$ and shot type $(d=1.30, p=.005)$ keywords. This finding provides an indication of the sources of information consciously attended to by skilled participants when constrained to anticipate based on contextual information alone, as would be the case under extreme time constraints, providing a novel contribution to the research literature. When less-skilled participants were making statements containing player positioning keywords such as " $I$ shot to the left hand side of my opponent, and I moved towards the net”, in the same trial skilled participants were making statements containing court geometry and shot type keywords such as "The space was on the left hand side for an angled passing shot”. It appears that the skilled participants are better able to use domain-specific information picked up from the relative movement and positioning of the two players to form meaningful evaluations about the availability of spaces and angles between players and the resultant potential shot selections of the opponent, as opposed to merely monitoring the players' positioning on the court. Furthermore, while the type of shot being played (e.g., forehand lob) is not immediately apparent from the visual display as the players' bodies and rackets are not visible, skilled participants appear to infer the type of shot being hit based on the information presented. Knowledge of the type of shot usually hit from a particular position on the court may allow the skilled players to more effectively evaluate the situation and interpret the tactical intentions of the opponent. 


\section{Gaze Data}

Search Rate

We hypothesized based on previous research on anticipation in tennis (e.g., Ward et al., 2002; Williams et al., 2002), that no differences would be found in search rate between groups and the differences would, in contrast, exist in the amount of time participants spent fixating on particular areas of the display. Pairwise comparisons revealed that skilled participants fixated on significantly fewer of the six potential fixation locations per trial than less-skilled participants ( $M=4.15, S E=.13$ vs. $M=4.67, S E=.20, d=1.04, p=.022$ ). No differences were found between groups for mean number of fixations or mean fixation duration. The data showing that skilled participants fixated on significantly fewer areas of the display per trial potentially indicate that skilled participants employed a more selective and, as such, more efficient gaze behavior than less-skilled participants (Williams \& Davids, 1998). However, it must be noted that while the effect size of this difference is large, the between-groups difference is small (approximately half of a fixation location per trial). We therefore draw conclusions from these data tentatively.

\section{Percentage Viewing Time}

Mean percentage viewing time and standard errors are presented in Figure 6. ANOVA revealed a significant Group $\times$ Fixation Location interaction, $F(3.10,49.54)=3.98, p=.012, \eta_{\mathrm{p}}{ }^{2}=.20$, and a main effect of Fixation Location, $F(3.10,49.54)=17.37, p<.001, \eta_{\mathrm{p}}{ }^{2}=.52$. Bonferroni-adjusted pairwise comparisons revealed that participants fixated the ball flight more than any other area. As hypothesized, differences were observed between groups for the amount of time spent fixating on the various features of the display. Pairwise comparisons revealed significant differences between groups for time spent fixating Ball Flight and Opponent areas of interest, with skilled participants fixating Ball Flight for a greater percentage of time $(M=42.55 \%, S E=3.63$ vs. $M=24.07 \%, S E=4.58, d=$ $1.50, p=.003$ ) than less-skilled participants. It is important to reiterate that we can only infer from gaze data the information participants may be processing from the visual display. It is possible that the skilled participants fixated for a greater amount of time on ball flight because they perceived this to be 
the most useful form of information available. However, based on previous findings (e.g., Ripoll et al., 1995; Vaeyens et al, 2007), it is possible that they are strategically anchoring gaze on the ball flight to more effectively extract and process pertinent information about the movement of the players relative to each other and the ball. Anchoring gaze on the ball flight may have allowed the skilled participants to more effectively extract informative cues arising from the relative movement and subsequent positions of the players through the use of peripheral vision. While skilled participants fixate on ball flight for a greater amount of time than any other area of the display, this attention to ball flight information is not evident in their verbal reports, in which ball flight is referred to comparatively little. While skilled participants are fixating for greater amounts of time on ball flight in this task than any other area, they may be concurrently attending to other, more important information from the display (cf., Williams \& Davids, 1999). These data, in conjunction with the verbal report data, provide an initial indication of the sources of contextual information skilled tennis players fixate on and attend to when anticipating based on contextual information alone.

$<<$ Insert Figure 6 here $>>$

To summarize, this experiment reaffirmed the importance of contextual information in anticipation. We demonstrated that skilled tennis players process contextual information differently to, and in essence, more effectively than less-skilled players. In support of LTWM theory (Ericsson \& Kintsch, 1995), skilled participants were shown to make more evaluation statements which are indicative of skilled participants more advanced domain-specific memory representations. The verbal reports of skilled participants were more detailed and varied relative to domain-specific keywords when compared with less-skilled counterparts. Furthermore, gaze behaviors differed between skill groups, with skilled participants fixating on the ball for greater amounts of time than the less-skilled participants. We tentatively proposed that skilled participants employed a visual anchoring strategy to more effectively extract pertinent information from the display through the use of peripheral vision.

\section{General Discussion}


We examined the ability of skilled and less-skilled tennis players to anticipate the depth and direction of an opponent's shot when postural information was either retained or omitted from the visual display, such that participants were constrained to anticipate based on the sequential relative movements of the players and the ball flight alone, or this information as well as postural information. We provided a novel contribution, investigating whether or not participants could anticipate effectively based on contextual information alone, as would be the case when constrained to anticipate in advance of pertinent postural cues becoming available due to extreme time constraints. We hypothesized, based on previous research (Abernethy et al., 2001; Triolet et al., 2013), that participants would be able to use this form of contextual information to anticipate effectively, and that the findings would indicate the extent to which they could do so relative to when postural information was also available. Moreover, we expected skilled participants to be able to use this information more effectively than their less-skilled counterparts (Abernethy et al., 2001). We further aimed to contribute to the development of psychological theory by investigating the perceptual-cognitive processes underlying anticipation when constrained to anticipate based on contextual information alone. We hypothesized, based on LTWM theory (Ericsson \& Kintsch, 1995), that skilled participants would make more evaluation and prediction statements which would be indicative of more advanced domain-specific memory representations and that skilled participants' gaze behavior would differ to less-skilled participants, in relation to the amount of time spent viewing the various features of the display (Ward et al., 2002; Williams et al., 2002).

First, both groups of tennis players were able to anticipate at levels greater than chance when constrained to anticipate based on contextual information alone, with skilled and less-skilled groups recording a mean combined judgment score of 60.30 and $47.00 \%$ over the two experiments respectively. These high judgment accuracy scores provide a clear indication of the importance of this form of contextual information in anticipation. This finding is novel since previously researchers have only been able to infer that contextual information may be used in isolation to anticipate effectively since postural information has always been available to participants (e.g., Abernethy et al., 2001; Triolet et al., 2013). We report the first attempt to remove postural information from the display such 
that the extent to which performers can use contextual information to anticipate could be more directly examined.

Second, our findings illustrate that while contextual information in the form of the sequential relative movement of the players and the ball flight is an important source of information that can be used to anticipate effectively by skilled and less-skilled performers, the accuracy of participants’ anticipation judgments increases substantially when they are also provided with postural information from the opponent, revealing an additive effect of both sources of information. Loffing et al. (2011) found an effect in the opposite direction (i.e., that when tennis players were provided with contextual information in the form of court positioning in addition to postural information, the accuracy of their judgments increased in comparison with when postural information was presented in the absence of contextual information). It is likely that the information upon which performers base their anticipation judgments in a real-world task is situation dependent (Roca et al., 2013) and that they conceivably opt for the most reliable source of information available. The findings of Triolet et al. (2013) indicate that in professional tennis, when players feel anticipation is necessary to avoid losing the point, they may only be able to wait for postural information to become available on a small number of instances due to the extreme time constraints involved. It is therefore likely, and it seems strategically viable, that players continuously process contextual information to inform potential anticipation judgments throughout rallies, acting on these advanced judgments in the case of severe time constraints, and calling upon more reliable postural information from the opponent, if the time taken to reach the opponent's shot is predicted to be less than it would take for the ball to pass the player.

Third, we have demonstrated how skilled tennis players more effectively process contextual information to anticipate with greater accuracy than their less-skilled counterparts. Skilled players appear to more effectively extract pertinent contextual information picked up from the visual display. This information may act as a retrieval cue, which is activated to retrieve task relevant information from LTM, resulting in more effective evaluation of the presented information and potential outcomes of the opponent's upcoming shot. While these data provide an indication of how skilled performers process contextual information in the absence of postural information, LTWM theory predicts that 
when task-relevant options exist, skilled performers would be expected to generate a greater number of task relevant options than less-skilled performers (Ward et al., 2013). An interesting line of research would therefore be to investigate how contextual and postural information interact with respect to option generation, as this has the potential to provide useful information for the design of testing and training protocols. It is possible though, that in the competitive environment skilled performers would generate more task-relevant options than less-skilled performers far in advance of the event based on the contextual information available (information that in absence of postural information can be used to anticipate effectively), and that the perceived feasible options would become fewer but more reliable in the build-up to the event due to the availability of pertinent postural information.

Although we have found that contextual information picked up in the form of the sequential relative movements of the players and the ball flight in the lead-up to the event, along with the resulting court positioning of the players at the moment of the event is a useful source of information which can be exploited by both skilled and less-skilled tennis players, we do not yet know what information specifically participants use to inform their judgments. For example, the gaze data indicated that skilled participants fixated for longer on the ball flight than their less-skilled counterparts, whereas the verbal report data indicated that skilled participants attended more to court geometry and shot type than their less-skilled counterparts. While it is possible that the ball flight is the most important and informative source of information available from the display, it is possible the relative positioning of the two players at the moment of racket-ball contact may provide the most useful information. In a similar way to how researchers manipulated point-light displays to determine the most informative sources of biological motion information picked up from an opponent (e.g., Cañal-Bruland, van Ginneken, van der Meer, \& Williams, 2011; Huys, Cañal-Bruland, Hagemann, Beek, Smeeton, \& Williams, 2009), there is a need for further research which manipulates the visual display to facilitate evaluation of the role and importance of the various sources of contextual information contained within rallies. Such an investigation would not only enhance our knowledge of perceptual-cognitive expertise but enable recommendations to be made as to what information 
developing players should attend to in anticipation training programs. Moreover, while researchers have demonstrated the effectiveness of anticipation training programs in both laboratory and fieldbased settings (Abernethy, Schorer, Jackson, \& Hagemann, 2012; Williams et al., 2002), only one study to our knowledge has trained participants to use contextual information when anticipating (Mann et al., 2014).

In recent years there has been a shift towards, and a call for, more research investigating the multidimensional nature of anticipation (Cañal-Bruland \& Mann, 2015). For example, Roca et al. (2013) provided an illustration of how the perceptual-cognitive skills and processes underlying anticipation interact as a function of the unique task constraints placed on the performer. The number of research studies investigating how contextual information contributes to anticipation is increasing (e.g., Crognier \& Féry, 2005; McRobert et al., 2011) and the work of Triolet et al. (2013) has provided us with behavioral evidence of the use of contextual information in a real-world setting. The present paper not only adds to a growing body of work in this area, but through the use of a novel methodological approach, and multiple process-tracing measures has been able to determine with much more certainty, if and how, performers utilize and process contextual information to inform their anticipation judgments.

The viewing perspective employed in this study may be considered to be a limitation in the study design as footage does not replicate the first person viewing perspective normally experienced in tennis. However, to examine the importance of contextual information presented as the sequential relative movement of players and the ball flight in the lead up to the event, it was necessary for participants to be able to see both players and how the velocities of their movements and positions on the court changed relative to the ball and each other throughout the rally. Additionally, while the third person perspective used is not that which would be experienced in a match situation, the relevant information presented is the same as that which would be available in a match (e.g., player positioning/velocity of movement/previous shots hit) albeit in a different form. Moreover, we report significant differences in anticipation across skill groups providing construct validity for the approach. On a similar point, while our gaze behavior data may not exactly replicate those which would be 
reported in an on-court setting (e.g., Mann, Farrow, Shuttleworth \& Hopwood, 2009), our findings suggest that skilled participants employed more effective gaze behaviors than their less-skilled counterparts. Furthermore, participants fixated on the player on the near side of the screen a mere $5.15 \%$ of total viewing time and only mentioned the near player approximately once per trial in their verbal reports, meaning that only a small amount of time was spent fixating on and attending to the one element of the display that would not be visible in a real-world scenario.

Due to technological limitations, there were differences between the normal video and animated display condition other than the mere omission of postural information in the animated condition. Other information such as the environmental surroundings, the crowd viewing the match, and the umpire were present in the normal video condition but not in the animated condition and we therefore cannot assume that the higher judgment accuracy scores reported in the normal video condition in comparison with the animated condition are solely a result of the additional postural information that participants were presented with. However, based on anticipation research to date (for a review, see Mann et al., 2007) we considered postural information to be the source of information with the most potential to inform participants' anticipation judgments that was present in the normal video but not the animated condition.

A further limitation of the present study is the relatively small sample size that compromises the statistical power of our analyses. The difficulty associated with recruiting very highly-skilled athletes, particularly in individual as opposed to team sports, is a common issue faced by researchers in this area. In practice it means that only larger effect sizes will attain statistical significance. In the experiments presented here, we were able to use the same number or more participants than in several related studies (e.g., Abernethy et al., 2001; Farrow \& Reid, 2012; McRobert et al., 2011) while being fewer than in others (e.g., Loffing et al., 2011; Loffing \& Hagemann, 2014). Accordingly, additional research is needed to determine whether comparisons that resulted in small or medium effect sizes are replicated, thereby allowing us to more confidently infer associated small or medium effects in the population. Conversely, statistically significant findings in the present study reveal large effects in 
comparisons between high-skilled players and less-skilled but experienced players rather than novices. They are therefore good candidates for having practical as well as statistical significance.

In conclusion, we used a novel experimental design to demonstrate that contextual information picked up from the sequential relative movement of the players and the ball flight in the lead up to the event effectively informs anticipation and that these judgments are made more accurate upon presentation of postural information. Due to the extreme time constraints involved in tennis, players may not always be afforded the opportunity to wait for postural information to become available. As such the ability to accurately anticipate based on contextual information in advance of such cues may prove to be a key contributing factor to expert performance in time-constrained domains. Furthermore, we found that skilled tennis players employed different gaze behaviors and more thoroughly evaluated this form of contextual information to anticipate more accurately than their less skilled counterparts. Finally, effective use of contextual information in advance of postural information may not only 'set the scene' for accurate anticipation when pertinent postural information becomes available, but crucially it may allow for highly accurate anticipation when extreme time constraints mean that waiting several moments longer is not a feasible option. 
References

Abernethy, B., Gill, D., Parks, S., \& Packer, S. (2001). Expertise and the perception of kinematic and situational probability information. Perception, 30(2), 233-252.

Abernethy, B., \& Russell, D. G. (1987). The relationship between expertise and visual search strategy in a racquet sport. Human Movement Science, 6(4), 283-319.

Abernethy, B., Schorer, J., Jackson, R. C., \& Hagemann, N. (2012). Perceptual training methods compared: The relative efficacy of different approaches to enhancing sport-specific anticipation. Journal of Experimental Psychology: Applied, 18(2), 143-153.

Abernethy, B., \& Wollstein, J. (1989). Improving anticipation in racquet sports. Australian Sports Commission. 15-18.

Abernethy, B., \& Zawi, K. (2007). Pickup of essential kinematics underpins expert perception of movement patterns. Journal of Motor Behavior, 39(5), 353-367.

Abernethy, B., Zawi, K., \& Jackson, R. C. (2008). Expertise and attunement to kinematic constraints. Perception, 37(6), 931-948.

Afonso, J., Garganta, J., McRobert, A. P., Williams, A. M., \& Mesquita, I. (2012). The perceptual cognitive processes underpinning skilled performance in volleyball: Evidence from eyemovements and verbal reports of thinking involving an in situ representative task. Journal of Sports Science \& Medicine, 11(2), 339-345.

Afonso, J., \& Mesquita, I. (2013). Skill-based differences in visual search behaviours and verbal reports in a representative film-based task in volleyball. International Journal of Performance Analysis in Sport, 13(3), 669-677.

Anderson, J. R. (1987). Methodologies for studying human knowledge. Behavioral and Brain Sciences, 10(3), 467-477. 
Buckolz, E., Prapavesis, H., \& Fairs, J. (1988). Advance cues and their use in predicting tennis passing shots. Canadian Journal of Sport Sciences, 13(1), 20-30.

Cañal-Bruland, R., \& Mann, D. L. (2015). Time to broaden the scope of research on anticipatory behavior: A case for the role of probabilistic information. Frontiers in Psychology, 6

Cañal-Bruland, R., van Ginneken, W. F., van der Meer, B. R., \& Williams, A. M. (2011). The effect of local kinematic changes on anticipation judgments. Human Movement Science, 30(3), 495503.

Catteeuw, P., Gilis, B., Wagemans, J., \& Helsen, W. (2010). Perceptual-cognitive skills in offside decision making: Expertise and training effects. Journal of Sport and Exercise Psychology, 32(6), 828-844.

Context. In Oxford Dictionaries Online Retrieved July 2, 2015, from http://www.oxforddictionaries.com/definition/english/context

Croft, J., Button, C., \& Dicks, M. (2010). Visual strategies of sub-elite cricket batsmen in response to different ball velocities. Human Movement Science, 29(5), 751-763.

Crognier, L., \& Féry, Y. (2005). Effect of tactical initiative on predicting passing shots in tennis. Applied Cognitive Psychology, 19(5), 637-649.

Crognier, L., \& Féry, Y. (2007). 40 ans de recherches sur l'anticipation en tennis: Une revue critique [40 years of research on anticipation in tennis: A critical review]. Movement \& Sport Sciences, 3(62), 9-35.

Eccles, D. W. (2012). Verbal reports of cognitive processes. In G. Tenenbaum, R.C. Eklund, \& A. Kamata (Eds.), Handbook of Measurement in Sport and Exercise Psychology. Champaign, IL: Human Kinetics. 
Ericsson, K. A., \& Kintsch, W. (1995). Long-term working memory. Psychological Review, 102(2), 211-245.

Ericsson, K. A., \& Kirk, E. (2001). Instructions for giving retrospective verbal reports. Tallahassee, FL: Florida State University.

Ericsson, K. A., \& Simon, H. A. (1980). Verbal reports as data. Psychological Review, 87(3), 215.

Ericsson, K. A., \& Simon, H. A. (1993). Protocol analysis: Verbal reports as data (Rev.ed.). Cambridge, MA: Bradford books/MIT Press.

Farrow, D., \& Reid, M. (2012). The contribution of situational probability information to anticipatory skill. Journal of Science and Medicine in Sport, 15(4), 368-373.

Gillet, E., Leroy, D., Thouvarecq, R., \& Stein, J. (2009). A notational analysis of elite tennis serve and serve-return strategies on slow surface. Journal of Strength and Conditioning Research, 23(2), $532-539$.

Goulet, C., Bard, C., \& Fleury, M. (1989). Expertise differences in preparing to return a tennis serve a visual information-processing approach. Journal of Sport \& Exercise Psychology, 11(4), 382398.

Gray, R. (2002a). Behavior of college baseball players in a virtual batting task. Journal of Experimental Psychology: Human Perception and Performance, 28(5), 1131-1148.

Gray, R. (2002b). "Markov at the bat": A model of cognitive processing in baseball batters. Psychological Science, 13(6), 542-547.

Hick, W. E. (1952). On the rate of gain of information. Quarterly Journal of Experimental Psychology, 4(1), 11-26. 
Kunde, W., Skirde, S., \& Weigelt, M. (2011). Trust my face: Cognitive factors of head fakes in sports. Journal of Experimental Psychology: Applied, 17(2), 110-127.

Huys, R., Cañal-Bruland, R., Hagemann, N., Beek, P. J., Smeeton, N. J., \& Williams, A. M. (2009). Global information pickup underpins anticipation of tennis shot direction. Journal of Motor Behavior, 41(2), 158-170.

Jackson, R. C., \& Mogan, P. (2007). Advance visual information, awareness, and anticipation skill. Journal of Motor Behavior, 39(5), 341-351.

Jackson, R. C., Warren, S., \& Abernethy, B. (2006). Anticipation skill and susceptibility to deceptive movement. Acta Psychologica, 123(3), 355-371.

Jones, C., \& Miles, T. (1978). Use of advance cues in predicting the flight of a lawn tennis ball. Journal of Human Movement Studies, 4(4), 231-235.

Land, M. F., \& McLeod, P. (2000). From eye movements to actions: How batsmen hit the ball. Nature Neuroscience, 3(12), 1340-1345.

Loffing, F., \& Hagemann, N. (2014). On-court position influences skilled tennis players' anticipation of shot outcome. Journal of Sport \& Exercise Psychology, 36(1), 14-26.

Loffing, F., Stern, R., \& Hagemann, N. (2015). Pattern-induced expectation bias in visual anticipation of action outcomes. Acta Psychologica, 161, 45-53.

Loffing, F., Wilkes, T., \& Hagemann, N. (2011). Skill level and graphical detail shape perceptual judgments in tennis. Perception, 40(12), 1447-1456.

Mann, D. L., Schaefers, T., \& Cañal-Bruland, R. (2014). Action preferences and the anticipation of action outcomes. Acta Psychologica, 152, 1-9. 
Mann, D. L., Farrow, D., Shuttleworth, R., Hopwood, M., \& MacMahon, C. (2009). The influence of viewing perspective on decision-making and visual search behaviour in an invasive sport. International Journal of Sport Psychology, 40(4) 546-564.

Mann, D. L., \& Savelsbergh, G. J. P. (2015). Issues in the measurement of anticipation. In J. Baker \& D. Farrow (Eds.), Routledge handbook of sport expertise (pp.166-175). New York, NY: Routledge.

Mann, D. T. Y., Williams, A. M., Ward, P., \& Janelle, C. M. (2007). Perceptual-cognitive expertise in sport: A meta-analysis. Journal of Sport \& Exercise Psychology, 29(4), 457-478.

McPherson, S. L. (1999). Tactical differences in problem representations and solutions in collegiate varsity and beginner female tennis players. Research Quarterly for Exercise and Sport, 70(4), 369-384.

McPherson, S. L. (2000). Expert-novice differences in planning strategies during collegiate singles tennis competition. Journal of Sport and Exercise Psychology, 22(1), 39-62.

McPherson, S. L., \& Kernodle, M. (2007). Mapping two new points on the tennis expertise continuum: Tactical skills of adult advanced beginners and entry-level professionals during competition. Journal of Sports Sciences, 25(8), 945-959.

McRobert, A. P., Williams, A. M., Ward, P., \& Eccles, D. W. (2009). Tracing the process of expertise in a simulated anticipation task. Ergonomics, 52(4), 474.

McRobert, A. P., Ward, P., Eccles, D. W., \& Williams, A. M. (2011). The effect of manipulating context-specific information on perceptual-cognitive processes during a simulated anticipation task. The British Journal of Psychology, 102(3), 519-534.

Müller, S., \& Abernethy, B. (2012). Expert anticipatory skill in striking sports: A review and a model. Research Quarterly for Exercise and Sport, 83(2), 175-187. 
Navia, J. A., van der Kamp, J., \& Ruiz, L. M. (2013). On the use of situational and body information in goalkeeper actions during a soccer penalty kick. International Journal of Sport Psychology, 44(3), 234-251.

North, J. S., Ward, P., Ericsson, K. A., \& Williams, A. M. (2011). Mechanisms underlying skilled anticipation and recognition in a dynamic and temporally constrained domain. Memory (Hove, England), 19(2), 155-168.

Paull, G., \& Glencross, D. (1997). Expert perception and decision making in baseball. International Journal of Sport Psychology, 28(1), 35-56.

Ripoll, H., Kerlirzin, Y., Stein, J., \& Reine, B. (1995). Analysis of information processing, decision making, and visual strategies in complex problem solving sport situations. Human Movement Science, 14(3), 325-349.

Roca, A., Ford, P. R., McRobert, A. P., \& Williams, A. M. (2011). Identifying the processes underpinning anticipation and decision-making in a dynamic time-constrained task. Cognitive Processing, 12(3), 301-310.

Roca, A., Ford, P. R., McRobert, A. P., \& Williams, A. M. (2013). Perceptual-cognitive skills and their interaction as a function of task constraints in soccer. Journal of Sport \& Exercise Psychology, 35(2), 144-155.

Rowe, R., Horswill, M. S., Kronvall-Parkinson, M., Poulter, D. R., \& McKenna, F. P. (2009). The effect of disguise on novice and expert tennis players' anticipation ability. Journal of Applied Sport Psychology, 21(2), 178-185.

Singer, R. N., Williams, A. M., Frehlich, S. G., Janelle, C. M., Radlo, S. J., Barba, D. A., \& Bouchard, L. J. (1998). New frontiers in visual search: An exploratory study in live tennis situations. Research Quarterly for Exercise and Sport, 69(3), 290-296. 
Thomas, J. R., Nelson, J. K., \& Silverman, S. J. (2005). Research methods in physical activity (5 ${ }^{\text {th }}$ ed.). Champaign, IL: Human Kinetics.

Triolet, C., Benguigui, N., Le Runigo, C., \& Williams, A. M. (2013). Quantifying the nature of anticipation in professional tennis. Journal of Sports Sciences, 31(8), 820-830.

Vaeyens, R., Lenoir, M., Williams, A. M., Mazyn, L., \& Philippaerts, R. M. (2007). The effects of task constraints on visual search behavior and decision-making skill in youth soccer players. Journal of Sport \& Exercise Psychology, 29(2), 147-169.

Ward, P., Ericsson, K. A., \& Williams, A. M. (2013). Complex perceptual-cognitive expertise in a simulated task environment. Journal of Cognitive Engineering and Decision Making, 7(3), 231254.

Ward, P., Williams, A. M., \& Bennett, S. J. (2002). Visual search and biological motion perception in tennis. Research Quarterly for Exercise and Sport, 73(1), 107-112.

Ward, P., Williams, A., \& Ericsson, K. (2003). Underlying mechanisms of perceptual-cognitive expertise in soccer. Journal of Sport \& Exercise Psychology, 25, S136.

Williams, A., \& Davids, K. (1997). Assessing cue usage in performance contexts: A comparison between eye-movement and concurrent verbal report methods. Behavior Research Methods, Instruments, \& Computers, 29(3), 364-375.

Williams, A. M., \& Davids, K. (1998). Visual search strategy, selective attention, and expertise in soccer. Research Quarterly for Exercise and Sport, 69(2), 111-128.

Williams, A. M., \& Elliott, D. (1999). Anxiety, expertise, and visual search strategy in karate. Journal of Sport \& Exercise Psychology, 21(4), 362-375. 
Williams, A. M., Ford, P. R., Eccles, D. W., \& Ward, P. (2011). Perceptual-cognitive expertise in sport and its acquisition: Implications for applied cognitive psychology. Applied Cognitive Psychology, 25(3), 432-442.

Williams, A. M., Janelle, C. M., \& Davids, K. (2004). Constraints on the search for visual information in sports. International Journal of Sport and Exercise Psychology, 2(3), 301-318.

Williams, A. M., Ward, P., Knowles, J. M., \& Smeeton, N. J. (2002). Anticipation skill in a realworld task: Measurement, training, and transfer in tennis. Journal of Experimental Psychology: Applied, 8(4), 259-270. 


\section{Figures}

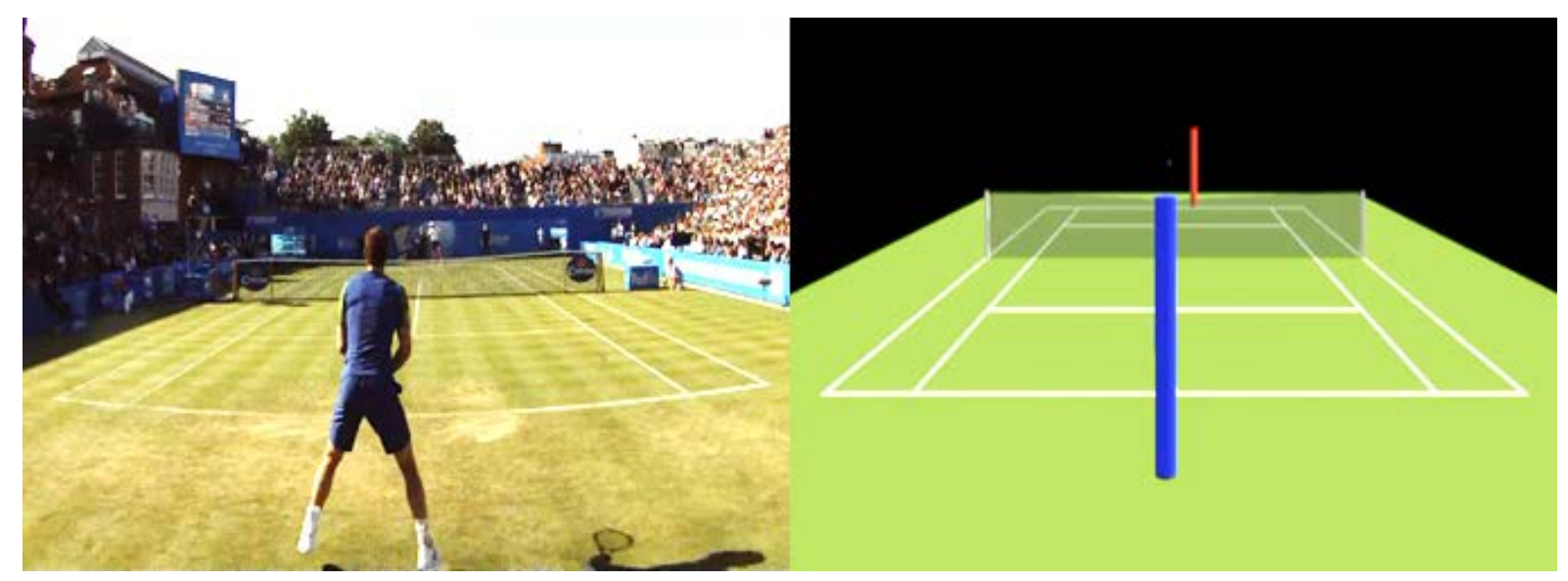

Figure 1. Normal video and animated display conditions. 


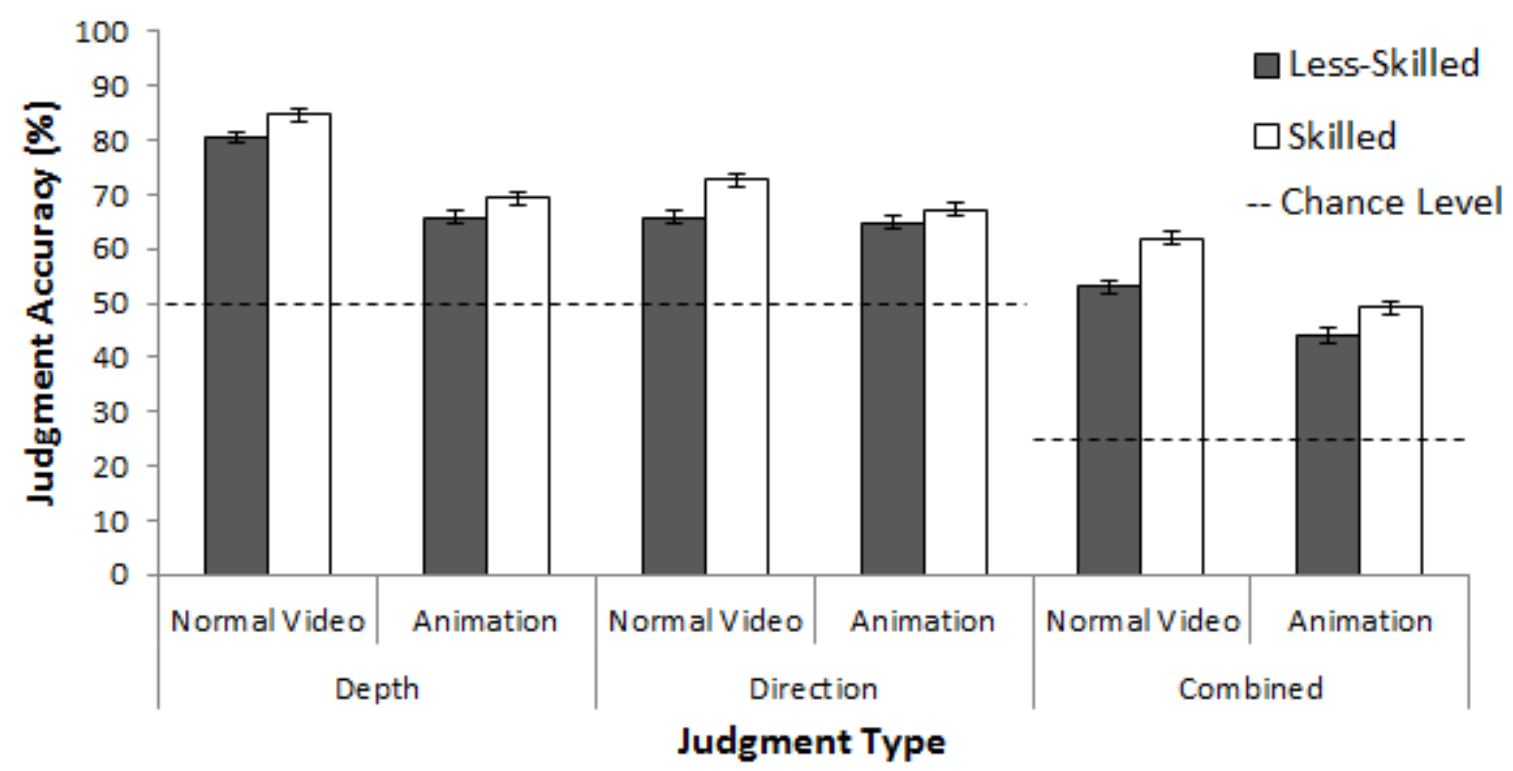

Figure 2. Judgment accuracy mean and standard error (90 normal video and 90 animated experimental trials) when making depth, direction, and combined anticipation judgments (Exp. 1). NV: normal video condition, ANI: animated condition. 


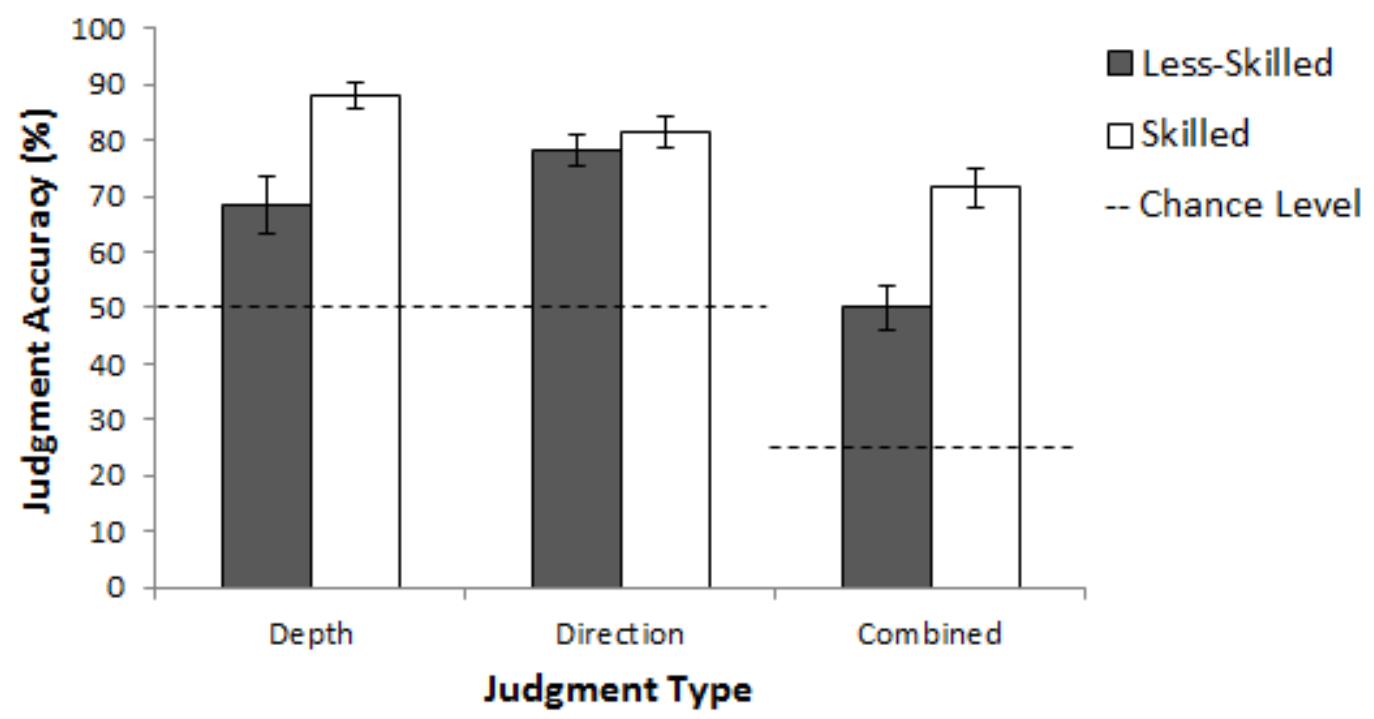

Figure 3. Judgment accuracy mean and standard error (20 experimental trials) when anticipating depth, direction and both depth and direction combined in the animated display condition (Exp. 2). 


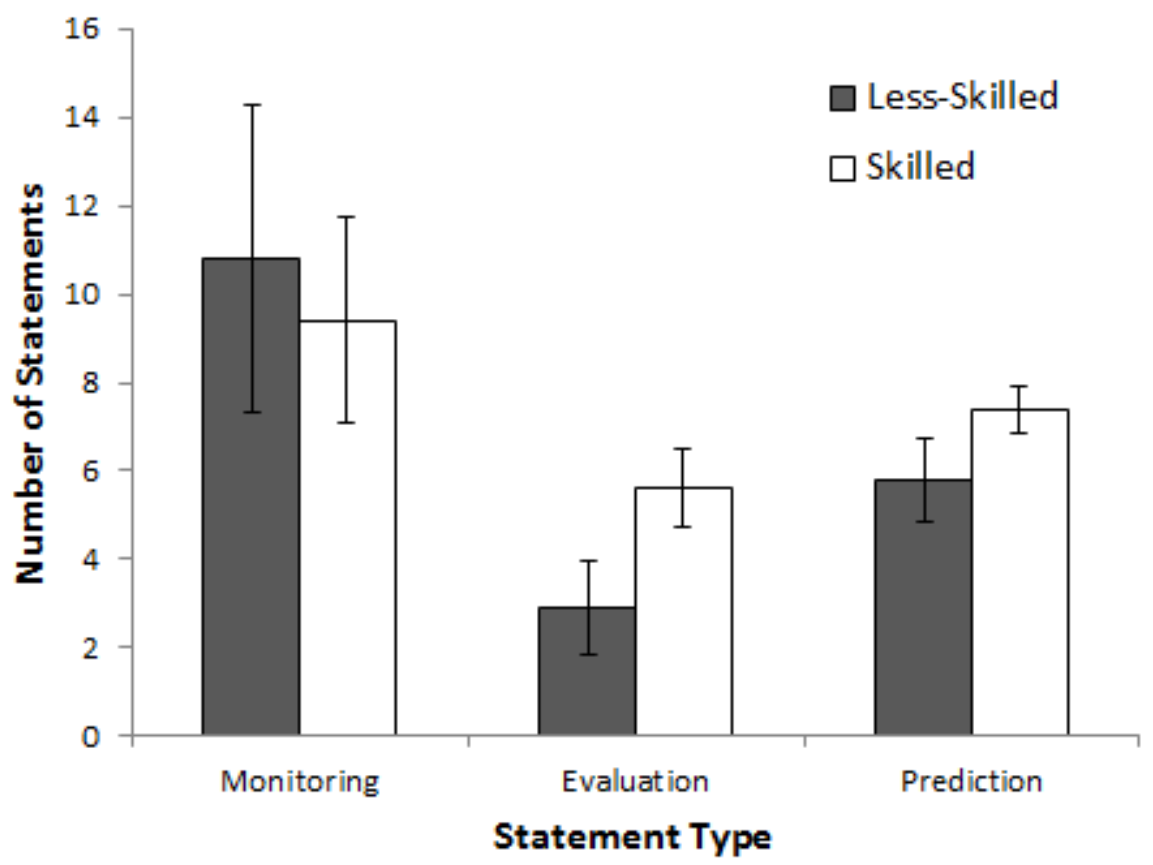

Figure 4. Mean and standard error of the number of verbal statements made by skilled and less-skilled participants (Exp. 2). 


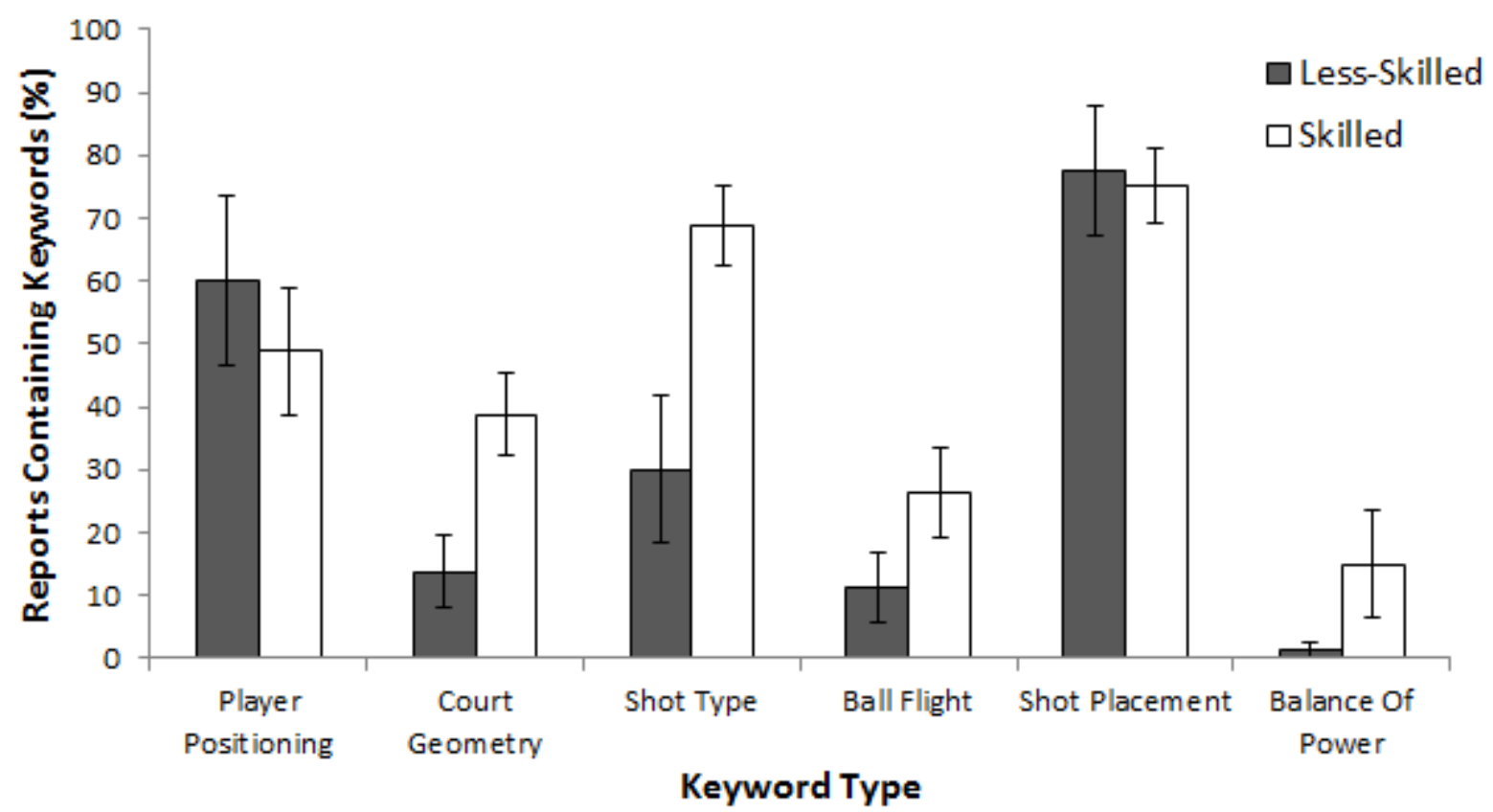

Figure 5. Mean and standard error of the percentage of the 8 reports containing keywords for skilled and less-skilled participants (Exp. 2). 


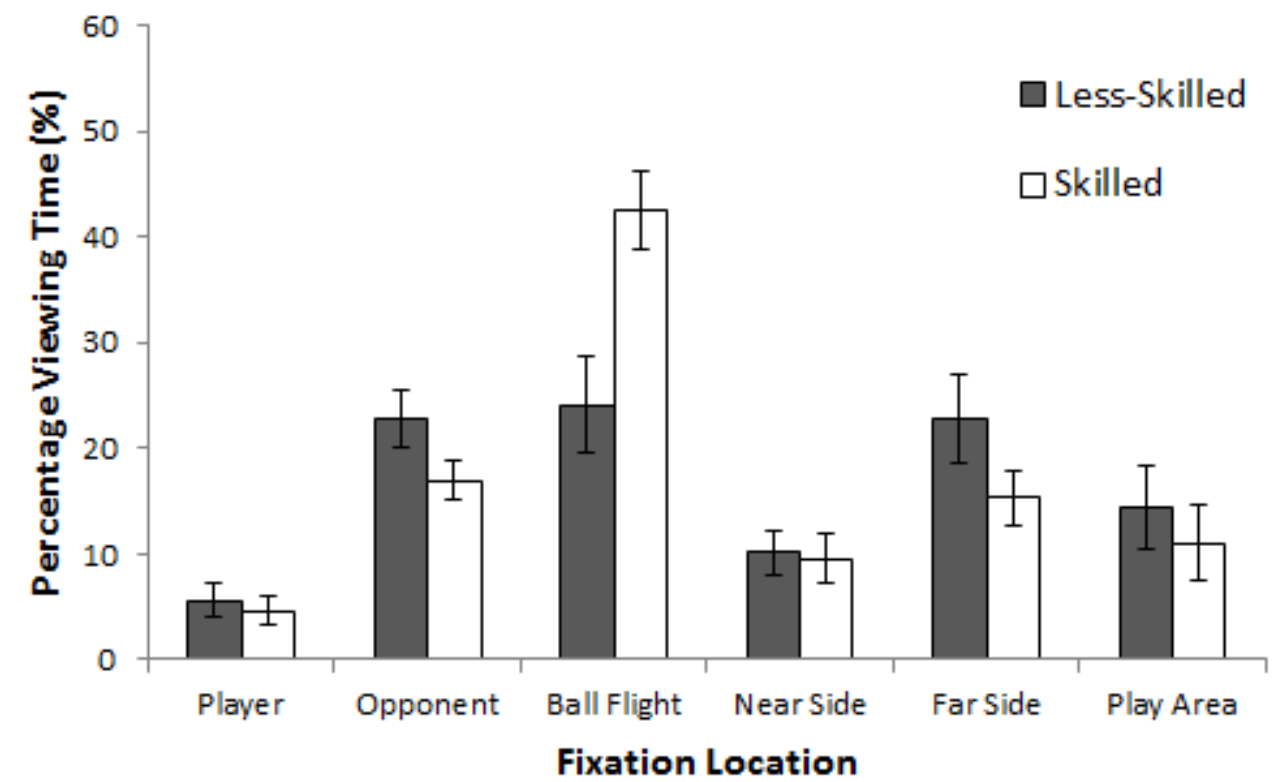

Figure 6. Mean percentage viewing time and standard error relative to fixation location of skilled and less-skilled participants (Exp. 2). 